\title{
To Achieve Circuit Robustness by Co-operation of FPAA and Embedded Microcontroller
}

\author{
György Györök, Tamás Orosz, Margit Makó, Tamás Treiber \\ Alba Regia University Center \\ Óbuda University \\ Budai Str. 45, H-8000 Székesfehérvár \\ \{gyorok.gyorgy, orosz.tamas, mako.margit\}@arek.uni-obuda.hu, treiber.tamas@gmail.com
}

\begin{abstract}
The cooperation of the analog and digital circuits and the embedded controllers as well as their industrial use and technical application have always been in the focus of our interest. In reconfigurable systems the effectiveness and quality of the analog circuit system can be maintained and modified by circuit or by changing the single element values. There are further advantages of the programmable analog circuit applications; more compact, more reliable, more flexible systems can be produced with better performance. It is especially beneficial if for programming we modify the function of a programmable analog circuit either giving a new topology or a new component parameter using the flexibility of the microcontroller.
\end{abstract}

\section{INTRODUCTION}

The reliability of electronic applications one side a part and manufacturing technology topic, on the other hand, there is the robustness of the used architecture. Greatness of robustness can define by equation (1);

$$
Q_{R_{\min }} \leq\left(\left.\frac{P_{\text {out }}}{P_{\text {in }}}\right|_{s \leqslant k}\right)<Q_{R_{\max }},
$$

where; $Q_{R_{\min }}$, and $Q_{R_{\max }}$ are the parameters of of the specified transfer function of electronic circuit, $s$ is a relevant environmental parameter, $k$ is a prognosticated limit of $s$.

The exact definition of robustness than the size of this paper, therefore, in a few aspects are highlighted.

On the electronic part market are any such circuit; Field Programmable Analog Array (FPAA), in-system Programmable Analog Circuit (ispPAC), Totally Reconfigurable Analog Circuit (TRAC), and programable system on chip (pSoC). In the realization of robust electronic circuit we will use the FPAA circuit, because it gives wide scale of servicing, and greatest flexibility in the relevant aspect.

In electrical application we can separate any different approach of circuit robustness [1] [2] [3]. Fig. 1. shows four main classification of a robust electronic system. The "change of circuit function and parametrical function" are synonymy of adaptivity, and wide range usable of an analog circuit [5].

The "failure of electrical part, failure of circuit" mostly question of, the electronic part technology, board manufacturing and testing [4].

The "altering of environmental conditions" in this case means the different physical parameters, so; temperature, humidity, moisture, vibration, acceleration, ... etc [5].

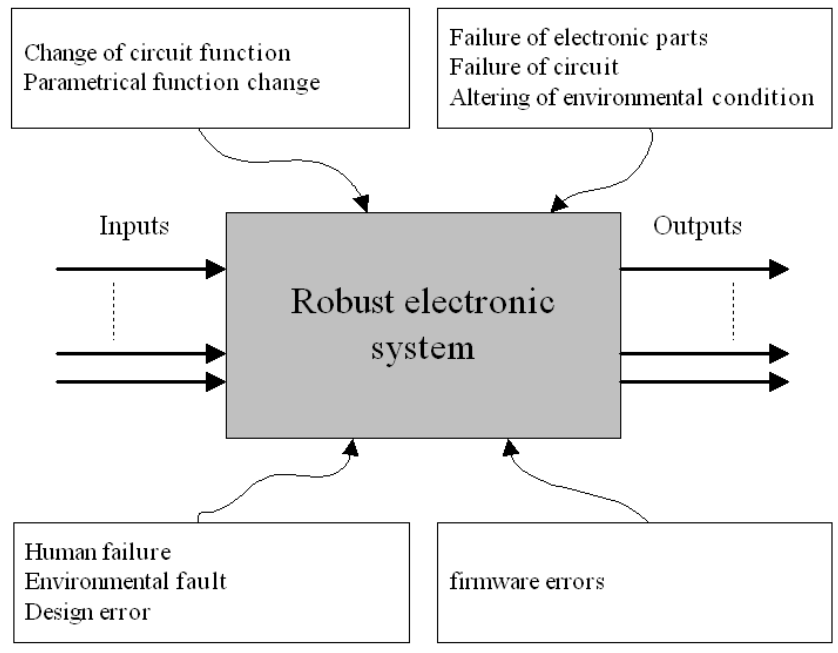

Fig. 1. Main aspects of a circuit robustness.

A box of Fig. 1 'human failure, environmental failure, design error" are a umbrella term, which in engineering practice is non handle, not to simple solve the handling of these [6] [7].

Firmware error suggests the every application contains a configuration memory or any stored firmware component[15].

The latter can provide an adaptive system in which is a cooperation between a microcontroller and a configurable analog circuit.

\section{ROBUST CIRCUIT SYSTEMS}

On the Fig. 2. can follow a classification of the adaptive hybrid systems. The traditional analog circuit (input devices, output amplifiers, power electronics, high frequency parts ...etc.) surrounds the filed programable analog array (FPAA), and an universal microcontroller $(\mu C)$ [8]. From environment circuit system gets input signals $S_{i n_{1}}-S_{i n_{m}}$, and gives to output signals $S_{\text {out }_{1}}-S_{\text {out }_{n}}$.

For these communication by each other are two surface; $\Sigma$ is the necessaries program strings for the configuration of FPAA topology and electronic part parameters. The $\Gamma$ is the stimulus which indicates to microcontroller a download a new 


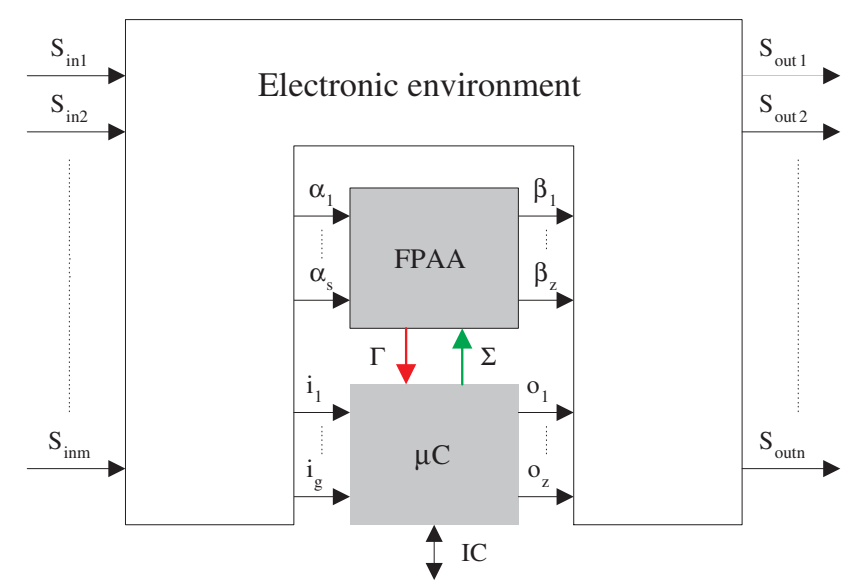

Fig. 2. Cooperation wit an embedded microcontroller and a field programable analog array.

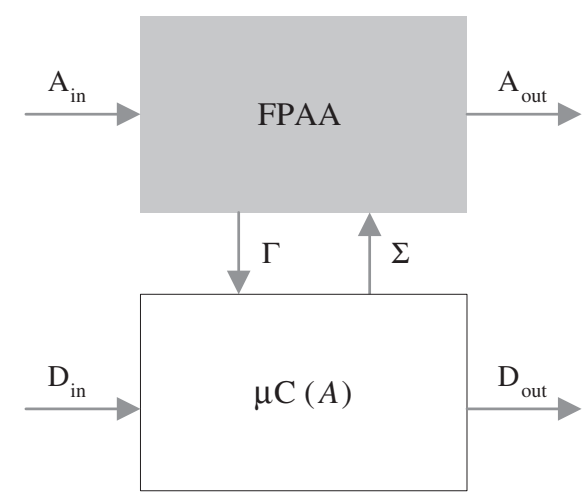

Fig. 3. Classification of hybrid core.

configuration into FPAA [9]. Through the interconnection IC surface we can fit this system to PC and other device [10] [11].

Fig. 3. shows an other approach of flexible hybrid core of proposed system.

The output function of an FPAA, used marking of Fig. 2 is (2) is;

$$
f_{F P A A}=\boldsymbol{f}(\bar{P}, n, \Gamma),
$$

where; $\bar{P}$ is parameter vector of the initial function of the FPAA, $n$ is the topology description of the currently used function of the FPAA, $\Gamma$ is feedback stimulus from analog circuit [9].

So we get the transfer function of FPAA circuit for the analog signals according equal (3);

$$
A_{\text {out }}=\mathbb{F}_{F P A A}\left(A_{\text {in }}\right),
$$

where; $\mathbb{F}_{F P A A}$ is a transfer function of FPAA circuit. For the digital signals write down in equal (4):

$$
D_{\text {out }}=\boldsymbol{g}\left(D_{\text {in }}\right) \text {, }
$$

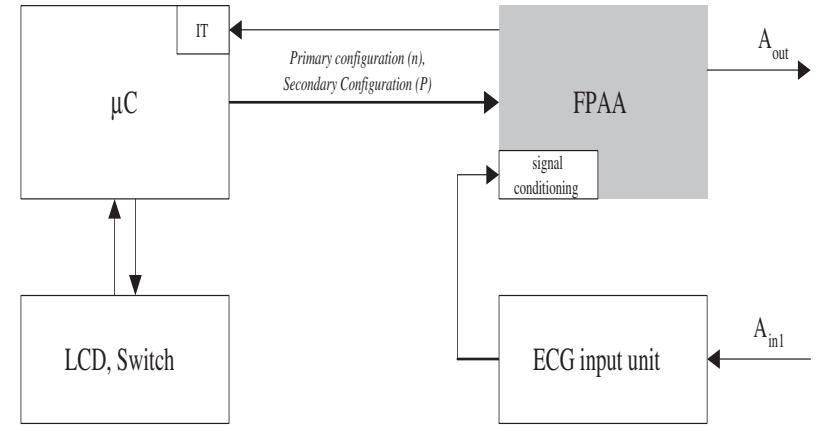

Fig. 4. Architecture of realized adaptive EEG input circuit as a robust electronic system.

where; $\mathbf{g}$ is a transfer function of microcontroller, according their firmware algorithm.

We have seen that an efficiently designed a arrangement a microcontroller and an FPAA cooperation a high degree of flexibility can be achieved.

\section{USING OF PROPOSED ROBUST ARRANGEMENTS}

We developed, according upper mentioned micro-controller FPAA connection such robust circuit, that useful for a medical electrocardiogram device (ECG) noise filtering and able to adapt to the variable heart frequency. This arrangement is shown in Fig. 4.

The realized robust system consist of a PIC16F887 type micro-controller and an Anadigm AN221E04 type Dynamically (Field) Programable Analog Array (dpASP). These devices communicate through serial peripheral interface (SPI) bus, where a micro-controller is the master and dpASP is the slave [12].

The bandpass filter was designed in AnadigmFilter software. The Primary configuration contain the topology and initialization parameters of the filter (Center Frequency, Pass Band Width, Stop Band Width, Pass Band Ripple, Pass Band Gain, Stop Band Attenuation) [9]. The implemented filter band pass width is $54 \mathrm{mHz}$, the stop band width is $216 \mathrm{mHz}$, and the stop band attenuation is $18 \mathrm{~dB}$. The size of Primary configuration is 132byte [13].

The dynamic reconfiguration is happen with state driven method. During the reconfiguration, the center frequency of bandpass filter is being stepped from $0,5 \mathrm{~Hz}$ to $4 \mathrm{~Hz}$ in 64 steps. This create 64 piece of 35 byte data array, which contains the necessary data for reconfiguration. The necessary data, for Primary and Dynamic configuration, can be generated by the AnadigmDesigner2 software [14] [15] [16].

After power up the stored program (Fig. 5) in the microcontroller initialize the Ports, the MSSP module for communication in SPI MASTER MODE0.The SPI clock is $2 \mathrm{MHz}$.

After that initialize the ECCP module in CAPCURE mode, with interrupt request on every rising edge of the incoming signal [17]. Thereafter display the power-up message on the LCD. Next send the primary configuration via the SPI interface, and if it was successful, then write the proper message on LCD display. Then the global interrupts are enabled. 


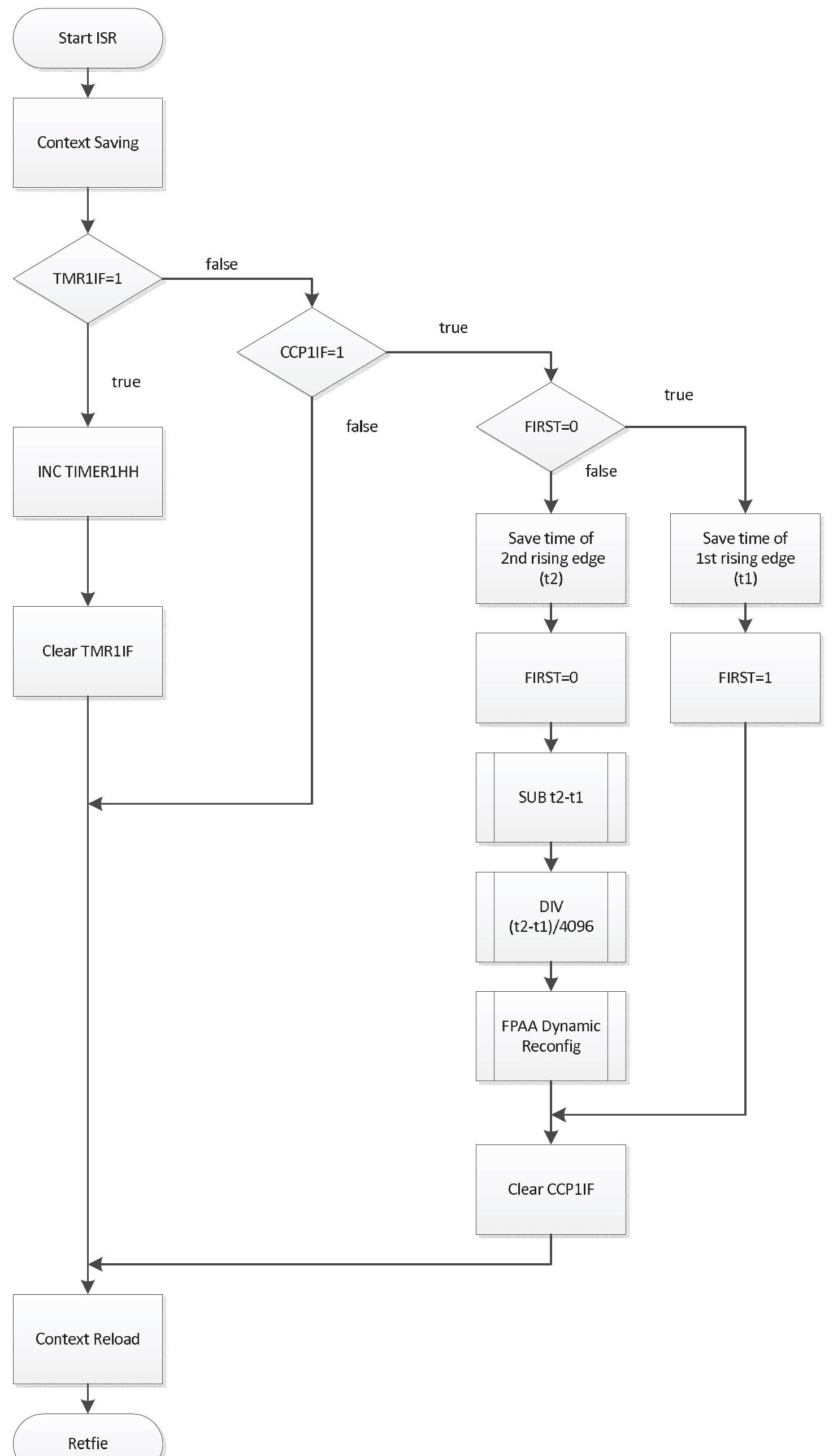




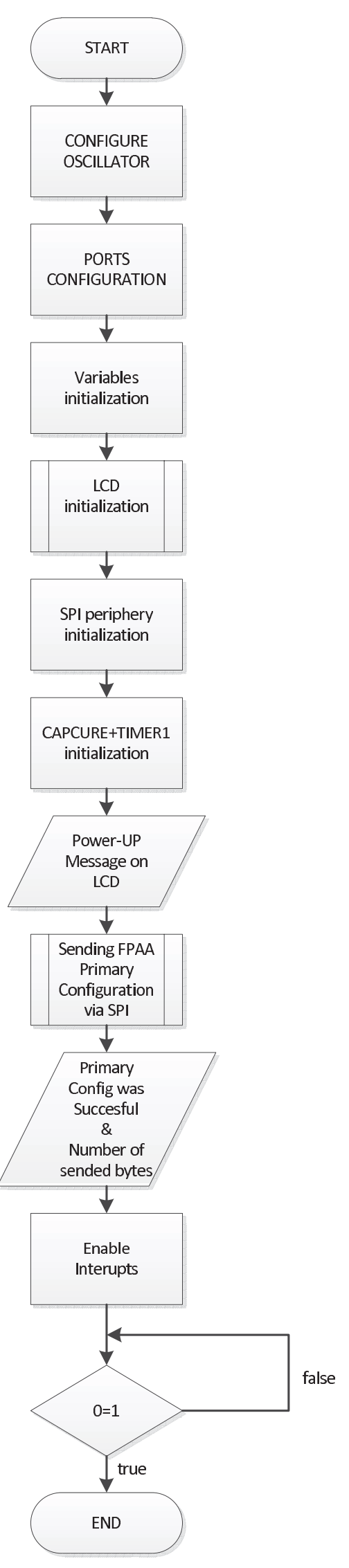

Fig. 5. Main flowchart of FPAA reconfiguration.

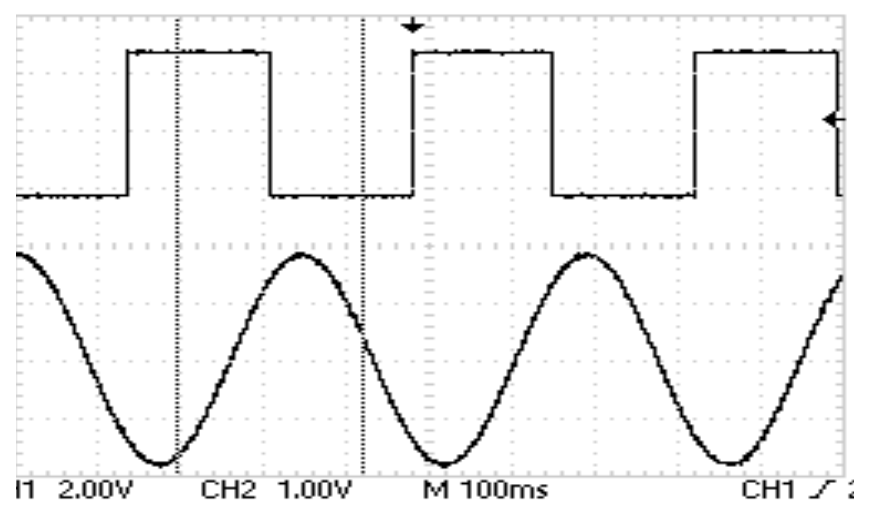

Fig. 8. Oscilloscope screen, bottom is the simulated EEG signal, upper by comparator modified square signal.

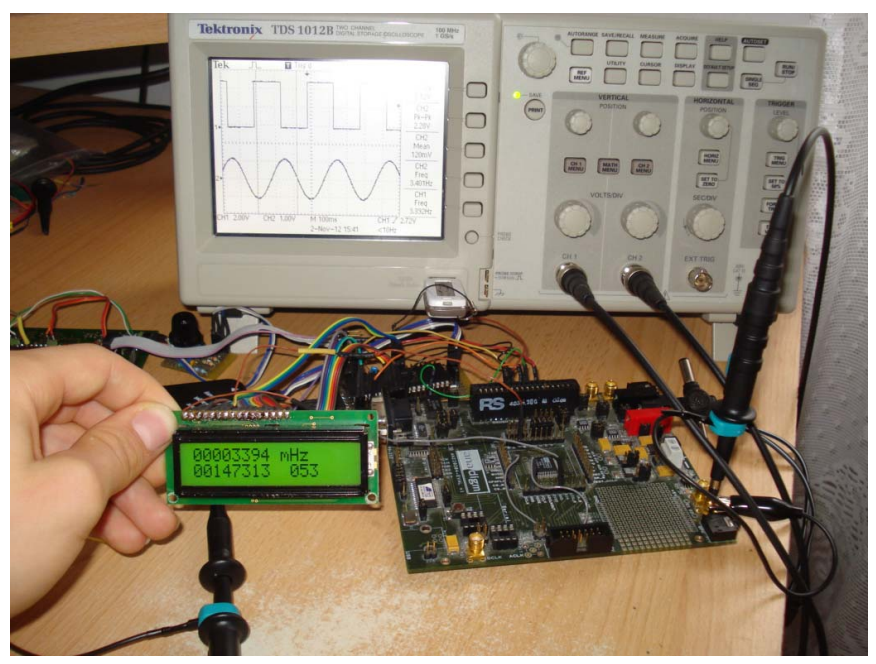

Fig. 9. Experimental environment, self developed MC-board, and a dpASP tool-kit. LCD shows the actual simulated heart-frequency.

Interrupt requests are generated by every rising edge of the CCP1 input signal (Fig. 6). For measuring a $0,5 \mathrm{~Hz}$ or a lower frequency signal, is necessary to extend the internal 16bit CAPCURE counter register to $24 \mathrm{bit}$, so the micro-controller at $16 \mathrm{MHz}$ clock frequency can to measure $0,5 \mathrm{~Hz}$ with $4 \mathrm{ppm}$ accuracy.

The measured frequency is tested in the first step, that is it included in the range from $0,5 \mathrm{~Hz}$ till $4 \mathrm{~Hz}$. If it is not, then the bandpass filter will not be reconfigured. If it is in the range, then it is determined by division, which dynamically reconfiguration data array should send out [18].

The primary and dynamic reconfiguration data is in the program memory of the micro-controller as Look-up tables. The realized circuit is seen on Fig. 7.

The measured frequency is in $\mathrm{mHz}$ dimension, the time elapsed between two rise edge, and the number of the reconfiguration data array, can be read from the LCD display, as an extra service for success developing (Fig. 8., 9.). 


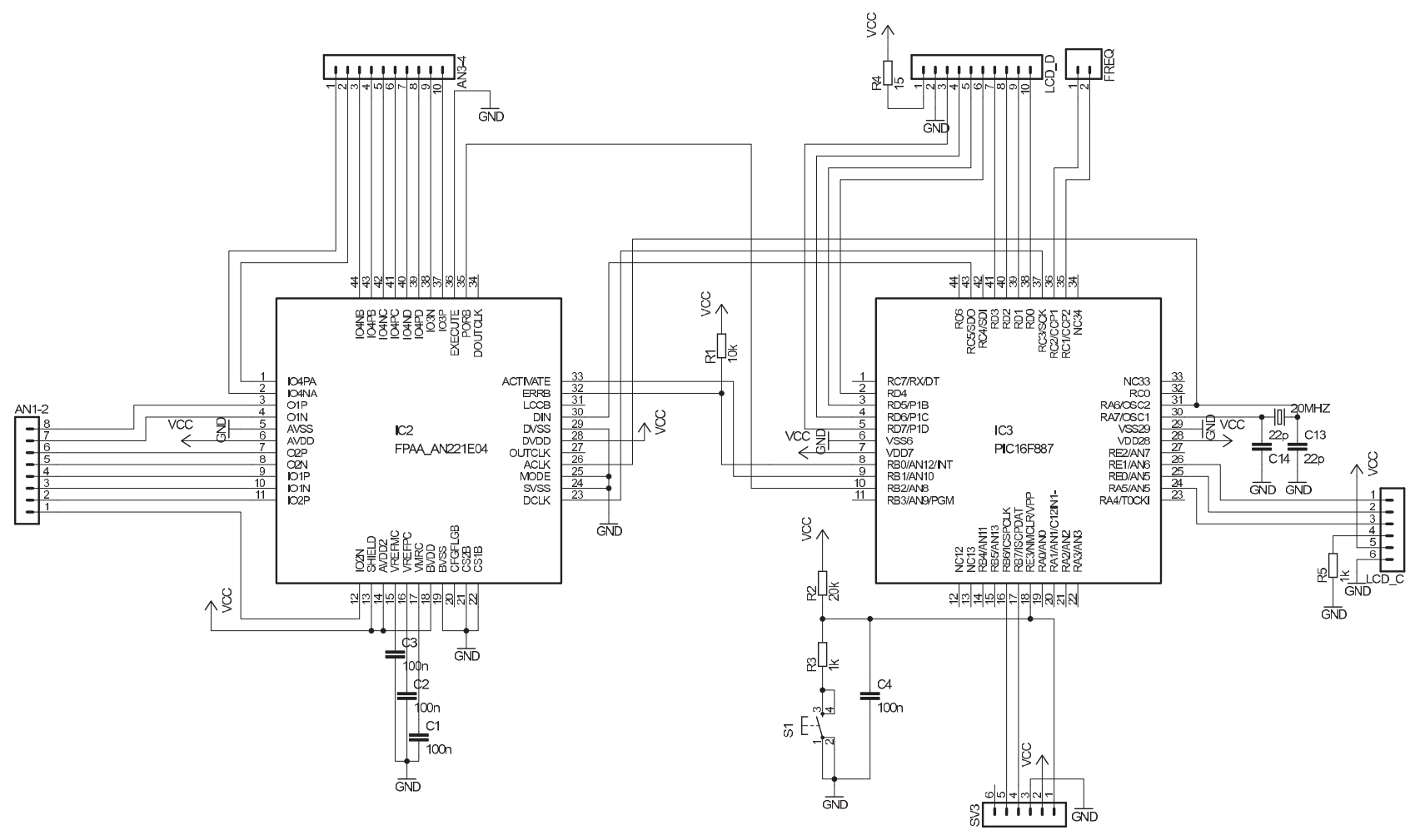

Fig. 7. Interconnection of micro-controller (PIC16F887) and dynamically programmable analog array (AN221E04).

\section{CONCLUSIONS}

In this paper, after a developed theoretical solution we have presented a useful real applications. Valuable part of our work is, that we have shown the electronic circuit and programming technology, and system components solutions.

The robust analog circuit solutions provide a consistent, high-level operation, by the cooperation of an embedded micro-controller and a programmable analog array circuit. By the mentioned solution the safety and effectiveness of the analog systems can be increased [10] [17].

We believe that the developed method can also be used in other areas [18] [19].

\section{REFERENCES}

[1] Gy. Györök. Self Organizing Analogue Circuit by Monte Carlo Method. LINDI 2007 International Symposium on Logistics and Industrial Informatics September 13-15, 2007 Wildau, Germany, ISBN 1-4244-1441-5, IEEE Catalog Number 07EX1864C, Library of Congress 2007930060, p. 34-37.

[2] Gy. Györök The FPAA Realization of Analog Robust Electronic Circuit. Proc. IEEE Internacional Conference on Computational Cybernetics: ICCC 2009. Palma de Mallorca, Spanyolorszg: pp. 1-5. Paper 10. (ISBN:978-1-4244-5311-5) 2009.11.26-2009.11.29.

[3] Gy. Györök A-class Amplifier with FPAA as a Predictive Supply Voltage Control. CINTI 2008 9th International Symposium of Hungarian Researchers on Computational Intelligence and Informatics, Budapest, Hungary. Budapest, Magyarorszg, 2008.11.06-2008.11.08. pp. 361-368.

[4] Gy. Györök, M. Makó. Self configuration Analog Circuits. XVIIth Kand conference 2006 ,In memoriam Klmn Kand” Budapest Tech Kand Klmn Faculty of Electrical Engineering, 12-14 January 2006, ISBN 9637154 426.
[5] Gy. Györök, M. Makó. Configuration of EEG Input-unit by Electric Circuit Evolution. INES 2005, 9th International Conference on Intelligent Engineering Systems, 2005 September 16-19, 2005 Cruising on Mediterranean Sea, ISBN 0-7803-9474-7, IEEE 05EX1202C.

[6] Gy. Györök. Self Configuration Analog Circuit by FPAA. 4th Slovakien-Hungarien Joint Symposium on Applied Machine Intelligence, 2006 January 20-21, Herlany, Slovakia, ISBN 9637154444 p. 34-37.

[7] Gy. Györök. The function-controlled input for the IN CIRCUIT equipment. IEEE-INES2004 Intelligent, Engineering Systems Conference, Cluj-Napoca, Romania, 2004 September 19-21, INES 2004, ISBN 973662-120-0, p. 443-446.

[8] Bandpass filter deging in Anadigm Filter. AnadigmDesigner2 User Manual.

[9] Sergyán, Sz. Content-Based Image Retrieval Using Automatically Determined Color Regions of Images. Proc. of 7th International Symposium on Applied Machine Intelligence and Informatics, Herl'any, Slovakia, January 30-31, 2009, pp. 41-45., ISBN 978-1-4244-3802-9, IEEE Catalog Number: CFP0908E-CDR

[10] Config data generation Anadigm App Note - 207 Application Note: State-Driven Control of a dpASP using a Microchip PIC.

[11] Gy. Györök. Programmable Analog Circuit in Reconfigurable Systems. 5th Slovakien - Hungarien Joint Symposium on Applied Machine Intelligence, 2007 January 25-26, Poprad, Slovakia, ISBN 978-963-7154-56-0, p. $151-156$.

[12] Tick, J. Potential Application of P-Graph-Based Workflow in Logistics. In: TAspects of Computational Intelligence: Theory and Applications: Revised and Selected Papers of the 15th IEEE International Conference on Intelligent Engineering Systems 2011, INES 2011. Heidelberg; London; New York: Springer Verlag, 2012. pp. 293-303. (Topics in Intelligent Engineering and Informatics) (ISBN:978-3-642-30667-9)

[13] Programmable System-on-Chip. http://www.cypress.com/?id=1353.

[14] Vámossy, Z. Delphi a gyakorlatban (Mintafeladatok megoldssal). ZAK Kiad, Bicske, 19972000, hrom kiads

[15] G. Hudoba, S. Berczi. The HUNVEYOR-project - a novel way of teaching Science and Physics HSCI2011, Proceedings of the 8th International Conference os Hands-on Science, Ljubljana, Sovenia, 2011. pp. 3-6., ISBN 978-989-95095-7-3,

[16] Tick, J. User Interface Redesign based on User Behavior Analyses. In: 
Proceedings of the ICCC 2003 IEEE International Conference on Computational Cybernetics. Konferencia helye, ideje: Sifok, Magyarorszg, 2003.08.29-2003.08.31. pp. 29-31.

[17] Dynamically Programable Analog Signal Processor. http://www.anadigm.com/solutions.asp.

[18] SPI connection between PIC and FPAA. AN121/221E04 User Manual.

[19] Mircea-Bogdan Rdac, Radu-Emil Precup, Emil M. Petriu, Stefan Preitl Experiment-based Performance Improvement of State Feedback Control Systems for Single Input Processes. In: Acta Polytechnica Hungarica Vol. 10, No. 1, 2013. pp.5-24. 